\title{
Tailward flow of energetic neutral atoms observed at Venus
}

\author{
A. Galli, ${ }^{1}$ M.-C. Fok, ${ }^{2}$ P. Wurz, ${ }^{1}$ S. Barabash, ${ }^{3}$ A. Grigoriev, ${ }^{3}$ Y. Futaana, ${ }^{3}$ M. Holmström, ${ }^{3}$ \\ A. Ekenbäck, ${ }^{3}$ E. Kallio, ${ }^{4}$ and H. Gunell ${ }^{5}$ \\ Received 31 January 2008; revised 11 April 2008; accepted 26 August 2008; published 2 December 2008.
}

[1] The Analyzer of Space Plasma and Energetic Atoms (ASPERA-4) experiment on Venus Express provides the first measurements of energetic neutral atoms (ENAs) from Venus. The results improve our knowledge on the interaction of the solar wind with a nonmagnetized planet and they present an observational constraint to existing plasma models. We characterize the tailward flow of hydrogen ENAs observed on the nightside by providing global images of the ENA intensity. The images show a highly concentrated tailward flow of hydrogen ENAs tangential to the Venus limb around the Sun's direction. No oxygen ENAs above the instrument threshold are detected. The observed ENA intensities are reproduced with a simple ENA model within a factor of 2, indicating that the observed hydrogen ENAs originate from shocked solar wind protons that charge exchange with the neutral hydrogen exosphere.

Citation: Galli, A., M.-C. Fok, P. Wurz, S. Barabash, A. Grigoriev, Y. Futaana, M. Holmström, A. Ekenbäck, E. Kallio, and H. Gunell (2008), Tailward flow of energetic neutral atoms observed at Venus, J. Geophys. Res., 113, E00B15, doi:10.1029/2008JE003096.

\section{Introduction}

[2] As a part of the Venus Express (VEX) scientific payload, the Analyzer of Space Plasma and Energetic Atoms (ASPERA-4) experiment, consisting of the Electron Spectrometer (ELS), the Ion Mass Analyzer (IMA), the Neutral Particle Detector (NPD) and the Neutral Particle Imager (NPI), is designed to study the plasma environment of Venus. It provides the first observations of energetic neutral atoms (ENA) from Venus, in a similar manner as the ASPERA-3 experiment on Mars Express (MEX) provided the first ENA observations of Mars [Futaana et al., 2006a, 2006b; Galli et al., 2006b]. Unlike the MEX spacecraft, VEX is also equipped with a magnetometer (MAG) allowing for a better characterization of the plasma environment.

[3] The first results of IMA, ELS, and MAG data from Venus have been published by Barabash et al. [2007b], Coates et al. [2008], Martinecz et al. [2008], and by Zhang et al. [2007]. The location and temporal variation of the plasma boundaries are found to be similar to the results derived from Pioneer Venus Orbiter (PVO) data [Martinecz et al., 2008]. Figure 1 shows the location of plasma boundaries derived in that study: at the bow shock the solar wind is slowed down to subsonic velocities, the magnetosheath between the bow shock and the inner plasma boundary is dominated by slow, heated up solar wind. The inner plasma boundary can either be defined as the

\footnotetext{
${ }^{1}$ Physikalisches Institut, Universität Bern, Bern, Switzerland.

${ }^{2}$ Geospace Physics Laboratory, NASA Goddard Space Flight Center, Greenbelt, Maryland, USA.

${ }^{3}$ Instituted för Rymdfysik, Kiruna, Sweden.

${ }^{4}$ Finnish Meteorological Institute, Helsinki, Finland.

${ }^{5}$ Department of Physics, West Virginia University, Morgantown, West Virginia, USA.

Copyright 2008 by the American Geophysical Union. 0148-0227/08/2008JE003096
}

boundary where the planetary ions start to dominate the plasma or as the boundary where the interplanetary magnetic field $B_{I M F}$ piles up around the ionosphere. The two different concepts are equivalent. In this paper we shall use the term "Induced Magnetosphere Boundary" (IMB) for the inner plasma boundary. It is close to the geometrical shadow behind Venus as is shown in Figure 1.

[4] Charge exchange between energetic ions from the plasma and neutral atoms in the exosphere produces ENAs. For a flux of monoenergetic protons $j_{\mathrm{p}}(r)$ that are neutralized along the line of sight (LOS) through atmospheric hydrogen with a density $n_{\mathrm{H}}(r)$ the ENA production equation reads

$$
j_{\mathrm{ENA}}=\sigma \int_{\mathrm{LOS}} d r n_{\mathrm{H}}(r) j_{\mathrm{p}}(r)
$$

with $j_{E N A}$ the ENA intensity in $\mathrm{cm}^{-2} \mathrm{sr}^{-1} \mathrm{~s}^{-1}$, and $\sigma$ the charge exchange cross section. For a proton-hydrogen reaction at $1 \mathrm{keV}$ energy $\sigma \approx 2 \times 10^{15} \mathrm{~cm}^{2}$, and $\sigma \approx 1 \times$ $10^{15} \mathrm{~cm}^{2}$ for $\mathrm{O}^{+}$-hydrogen charge exchange [Lindsay and Stebbings, 2005]. ENA measurements therefore reflect the plasma flux distribution as well as the neutral densities along the LOS of the sensor. The motivation of the ENA observations at Mars and Venus is to better understand the interaction of the solar wind with the neutral atmosphere of nonmagnetized planets. To interpret the observations, comparison with ENA models is needed. Such comparisons in turn constrain the uncertainties about the exospheric densities and the plasma populations implemented in the model.

[5] For Venus, so far only the ENA model predictions by Fok et al. [2004] and by Gunell et al. [2005] are available. For Mars, the list is longer. The ENAs originating from solar wind and magnetosheath protons were modeled by Holmström et al. [2002]. ENAs originating from planetary 


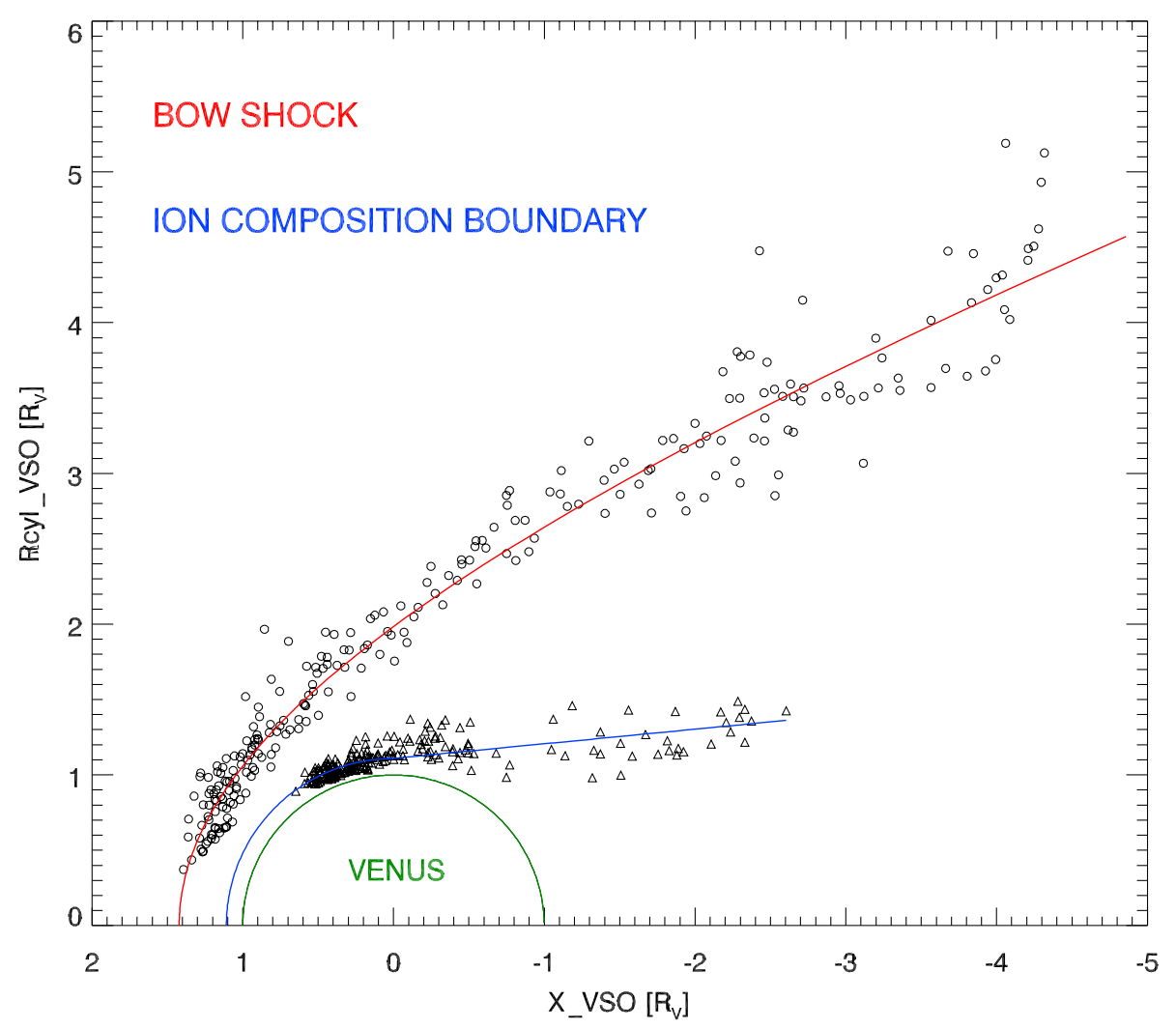

Figure 1. The two plasma boundaries around Venus, bow shock and Induced Magnetosphere Boundary (IMB), as measured with Analyzer of Space Plasma and Energetic Atoms/Ion Mass Analyzer (ASPERA-4/IMA) and electron spectrometer (ASPERA-4/ELS) during the first 5 months of observations [Martinecz et al., 2008]. The coordinates are given relative to the Venus Solar Orbital (VSO) reference frame in units of planetary radii $R_{\mathrm{V}}$. The $x$ axis is the Sun-Venus line $X_{\mathrm{VSO}}$, and the $y$ axis is the distance $\sqrt{Y_{V S O}^{2}+Z_{V S O}^{2}}$ to $X_{\mathrm{VSO}}$.

hydrogen [Lichtenegger et al., 2002] and from planetary oxygen [Barabash et al., 2002] were also investigated, but both for Mars and Venus the total ENA production is expected to be dominated by ENAs originating from solar wind protons. Because charge exchange hardly affects the energy of the fast particle, the typical energy of ENAs around Mars and Venus is predicted to be comparable to the $1 \mathrm{keV}$ of the solar wind. The ENA models also show that the ENA outflow originating from planetary ions is coaligned with the convective electric field $\mathbf{E}=-\mathbf{v}_{\mathrm{SW}} \times \mathbf{B}_{\mathrm{IMF}}$. On the other hand, the ENA outflow caused by solar wind protons is homogeneous around the planetary limb with respect to the Sun's direction, irrespective of the $B_{\mathrm{IMF}}$ direction. Gunell et al. [2006] show that this prediction does not change when an MHD or a hybrid code instead of an empirical code is implemented. The observations of the Martian ENAs made with MEX/NPD agree with the basic characteristics of the ENA models. The ENA emission of the dayside atmosphere and the tailward flow of ENAs from shocked solar wind are easily recognizable [Futaana et al., 2006a, 2006b; Galli et al., 2006b]. By analogy, we expect to see above the dayside of Venus solar wind ENAs that are scattered back from the atmosphere and a narrow ENA stream of shocked solar wind right above the subsolar point in the magnetosheath. On the nightside we expect to observe a tailward flow of ENAs, for which unperturbed solar wind, magnetosheath plasma, or accelerated planetary ions are possible parent ions. The latter parent population is the only one that also produces a measurable amount of oxygen ENAs.

[6] Because of the UV sensitivity of the NPD sensor, ENA measurements outside the Venus shadow have to be avoided in most cases. We will therefore concentrate on the tailward ENA flow observed when the spacecraft is inside the shadow. First examples of such measurements were published by Galli et al. [2008]. Here, we will provide a complete account of the nightside ENA observations. We will briefly describe the NPD sensor in section 2, before presenting the database that underlies this work (section 3). In section 4 we will show the global intensity images of tailward-flowing hydrogen ENAs; they will be compared to model predictions in section 5. The search for oxygen ENAs at Venus will be summarized in section 6 , followed by the conclusions in section 7 .

\section{NPD Instrument}

[7] NPD is one of the four sensors constituting the ASPERA-4 [Barabash et al., 2007a] experiment on ESA's VEX spacecraft (orbit insertion in May 2006). NPD is designed to measure hydrogen ENAs at energies between 

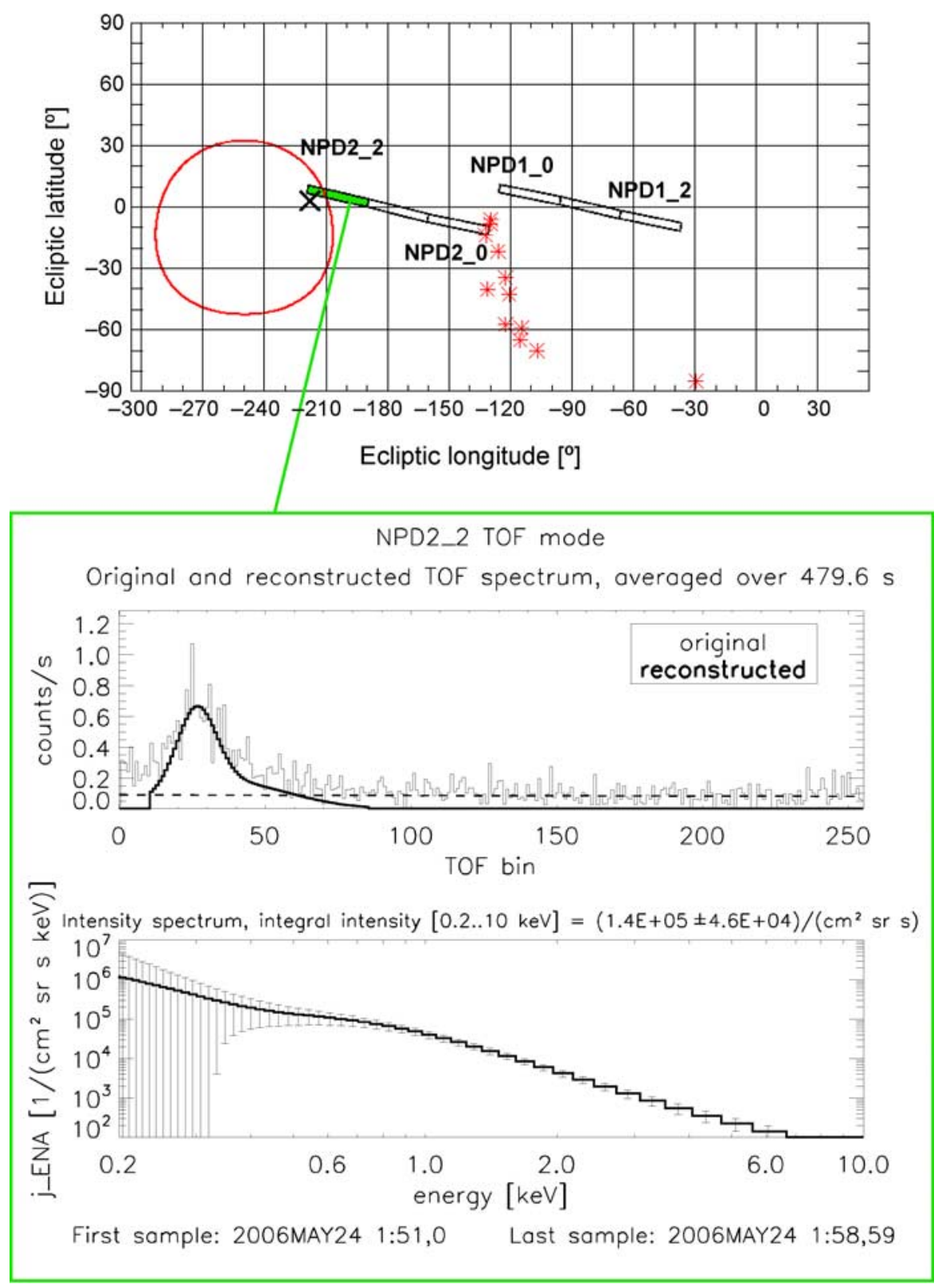

Figure 2. Typical energetic neutral atom (ENA) energy spectrum, integrated over $8 \mathrm{~min}$ of observation time in Venus eclipse on 24 May 2006. Top shows the spacecraft centered view. The black cross is the Sun's direction, the thick red line is the Venus limb. The red stars that cover the edge of the field of view (FOV) of sector NPD2 0 denote the limb of the solar panel. Middle and bottom show the time-of-flight (TOF) spectrum and the derived ENA intensity spectrum observed in channel NPD2_2 (see text for further explanations).

0.2 and $10 \mathrm{keV}$ and oxygen ENAs between 0.4 and $10 \mathrm{keV}$, using the time-of-flight (TOF) technique. Angular resolution is provided by having two NPD sensors (NPD1 and NPD2), each with three angular channels with a field of view $(\mathrm{FOV})$ of $30^{\circ} \times 5^{\circ}$ giving a total instantaneous FOV of $180^{\circ} \times 5^{\circ}$ [Barabash et al., 2007a]. We can distinguish between hydrogen and oxygen ENAs mainly because the velocities of hydrogen and oxygen ENAs of equal energy are very different. Only oxygen ENAs more energetic than several keV have TOF values that overlap with the ones of hydrogen ENAs between $0.2 \mathrm{keV}$ and $0.5 \mathrm{keV}$.
[8] Beside ENAs, NPD is also sensitive to UV photons. Observations with the Sun in the FOV therefore have to be avoided. Unfortunately, the dayside hydrogen exosphere of Venus proves to be too bright (more than $20 \mathrm{kR}$ Lyman- $\alpha$ [Bertaux et al., 1978]) for the NPD sensor as well.

[9] The spectrum reconstruction from raw data follows the same method described by Galli et al. [2006a]. As improvement over the first report on tailward ENAs observed at Venus [Galli et al., 2008] we have completed the database for observations inside the IMB and we have now incorporated the final laboratory calibration informa- 


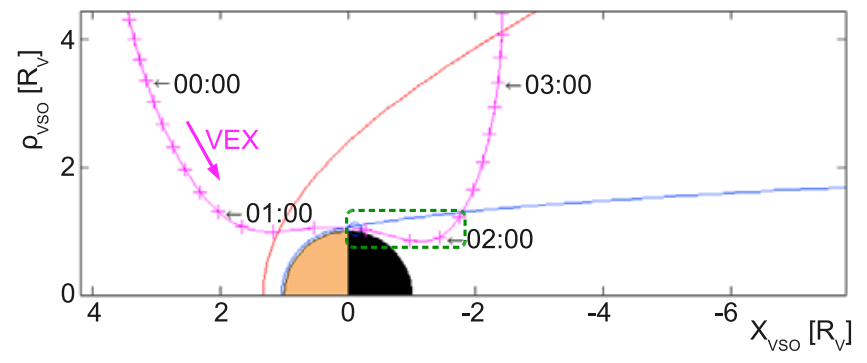

Figure 3. Venus Express orbit trajectory on 24 May 2006, plotted in the same coordinate system as Figure 1. The green rectangle highlights the time period $(30 \mathrm{~min}$ around $0200 \mathrm{UT}$ ) during which the spacecraft is inside the shadow of Venus.

tion for NPD, which became available recently [Grigoriev, 2007]. The instrument response, efficiency, and geometrical factors have remained the same within the error bars since our earlier publication [Galli et al., 2008].

\section{Database}

[10] The database of nightside observations includes all suitable ENA observations made inside the eclipse of Venus from orbit insertion until the end of 2007. We will not discuss observations for which the FOV was directed away from Venus, but up to now no signals above the detection threshold have been encountered under such circumstances.

[11] Observations with poor counting statistics or with other technical problems were excluded from further analysis. For instance, several of the six sectors of NPD may be obstructed by the solar panel, as is illustrated in Figure 2 by the red stars adjacent to the two sectors NPD2 0 and NPD1_0. To complicate things further, the ASPERA-4 main unit is mounted on a rotating platform. This allows for a faster coverage of the sky but drastically blurs the ENA signal for a given pointing direction. By default, the NPD FOV is rotated by $180^{\circ}$ every 32 seconds. The minimum integration time of two minutes for a FOV sector of $30^{\circ}$ therefore necessitates an observation time longer than $6 \times$ 2 minutes. In the meantime the spacecraft position relative to Venus may vary considerably.

[12] The following VEX orbits belong to the database of nightside NPD observations:

[13] 1. Eclipse season from 16 to 29 May 2006, 11 different suitable orbits. Detection threshold is $1 \times 10^{4} \mathrm{~cm}^{-2} \mathrm{sr}^{-1} \mathrm{~s}^{-1}$.

[14] 2. Eclipse season from 19 August to 3 September 2006, 5 suitable orbits. For this period the raw ENA intensities have been multiplied by a factor $4 \pm 2$ to compensate for a decrease in detection efficiency caused by an overexposure to UV light. Detection threshold is $4 \times 10^{4} \mathrm{~cm}^{-2} \mathrm{sr}^{-1} \mathrm{~s}^{-1}$.

[15] 3. Eclipse season from 27 November 2006 to 7 January 2007: never a reliable ENA signal above the detection threshold of several $10^{4} \mathrm{~cm}^{-2} \mathrm{sr}^{-1} \mathrm{~s}^{-1}$.

[16] 4. Eclipse season from 8 July to 16 August 2007. Detection threshold is several $10^{4} \mathrm{~cm}^{-2} \mathrm{sr}^{-1} \mathrm{~s}^{-1}$. All observations are taken into account that have suitably high count rates to synthesize a TOF spectrum. The ENA intensity derived from these measurements is to be understood only as a relative number to be multiplied with a yet unknown correction factor because the NPD sensor settings have been adjusted to make up for the decrease in detection efficiency. This gives us 12 different orbits from 8 July to 4 August 2007, and four further suitable orbits from 5 to 16 August 2007 when the end of this eclipse season approached.

[17] We have statistically evaluated (see section 5) only the data obtained during the first two eclipse seasons (16 different orbits), for which absolute intensities can be derived. All ENA intensities were integrated over the energy range from 0.2 to $10 \mathrm{keV}$, the uncertainty of the final value being 30\% typically. For analysis, all measurements were divided into intervals of two to ten minutes integration time, two minutes being enough only for very intense ENA signals. Moreover, we restricted the data evaluation to sector NPD2 2 because of the six NPD channels only this one was directed at the tailward ENA flow. All observations correspond to low solar activity.

\section{Results}

[18] Figure 2 shows a typical NPD observation of the tailward hydrogen ENA flow, obtained on 24 May 2006. The VEX orbit trajectory for that date is sketched in Figure 3. As for most observations in our database, the time window for NPD observations inside the Venus eclipse was roughly $30 \mathrm{~min}$ (green rectangle in Figure 3). Figure 2 (top) shows the spacecraft centered view for 0154:46 UT, when VEX was on the nightside of Venus at an altitude of $2900 \mathrm{~km}$. The thick red line indicates the Venus limb, the cross marks the Sun's position. Shown in black are the six NPD sectors, the red stars close to sectors NPD2_0 and NPD1_0 outline the solar panel. Figure 2 (middle) shows the original and the reconstructed TOF spectrum of the hydrogen ENAs (peak between TOF bin 10 and 100) measured in sector NPD2_0. The flat background in the TOF spectrum (dashed line) is caused by UV light. Figure 2 (bottom) shows the ENA intensity spectrum derived from the TOF spectrum in the energy range from 0.2 to $10 \mathrm{keV}$. The ENA intensity, integrated from 0.2 to $10 \mathrm{keV}$, calculates to $1.4 \times 10^{5} \mathrm{~cm}^{-2} \mathrm{sr}^{-1} \mathrm{~s}^{-1}$.

[19] The intensity spectrum shown in Figure 2 (bottom) is typical for the hydrogen ENA signals observed with NPD. The intensity spectrum shown in Figure 2 may be described as a two-component power law with a low-energy slope of $-1.8 \pm 0.2$, a rollover at $1.0 \pm 0.1 \mathrm{keV}$, and a slope at energies above $1.0 \mathrm{keV}$ of $-3.3 \pm 0.1$. Taking into account all ENA measurements from 2006 with a high signalto-noise ratio we find that most intensity spectra can be characterized by a two-component power law with a rollover between 0.4 and $2.0 \mathrm{keV}$. The median rollover lies at $1.1 \mathrm{keV}$. Unfortunately, for many measurement intervals poor counting statistics avoid a reliable reconstruction of the spectral shape of the ENA signal. Therefore, we do not use the shape of the measured spectrum as observational constraint for the ENA model. In section 5 we will illustrate the problem of interpreting measured energy spectra by showing a modeled ENA spectrum (see Figure 8). To make a quantitative comparison between observations and model, we will only use the integral ENA intensities because they are more reliable.

[20] We have synthesized images of the ENA intensities taking into account all measurement intervals that fulfill the 


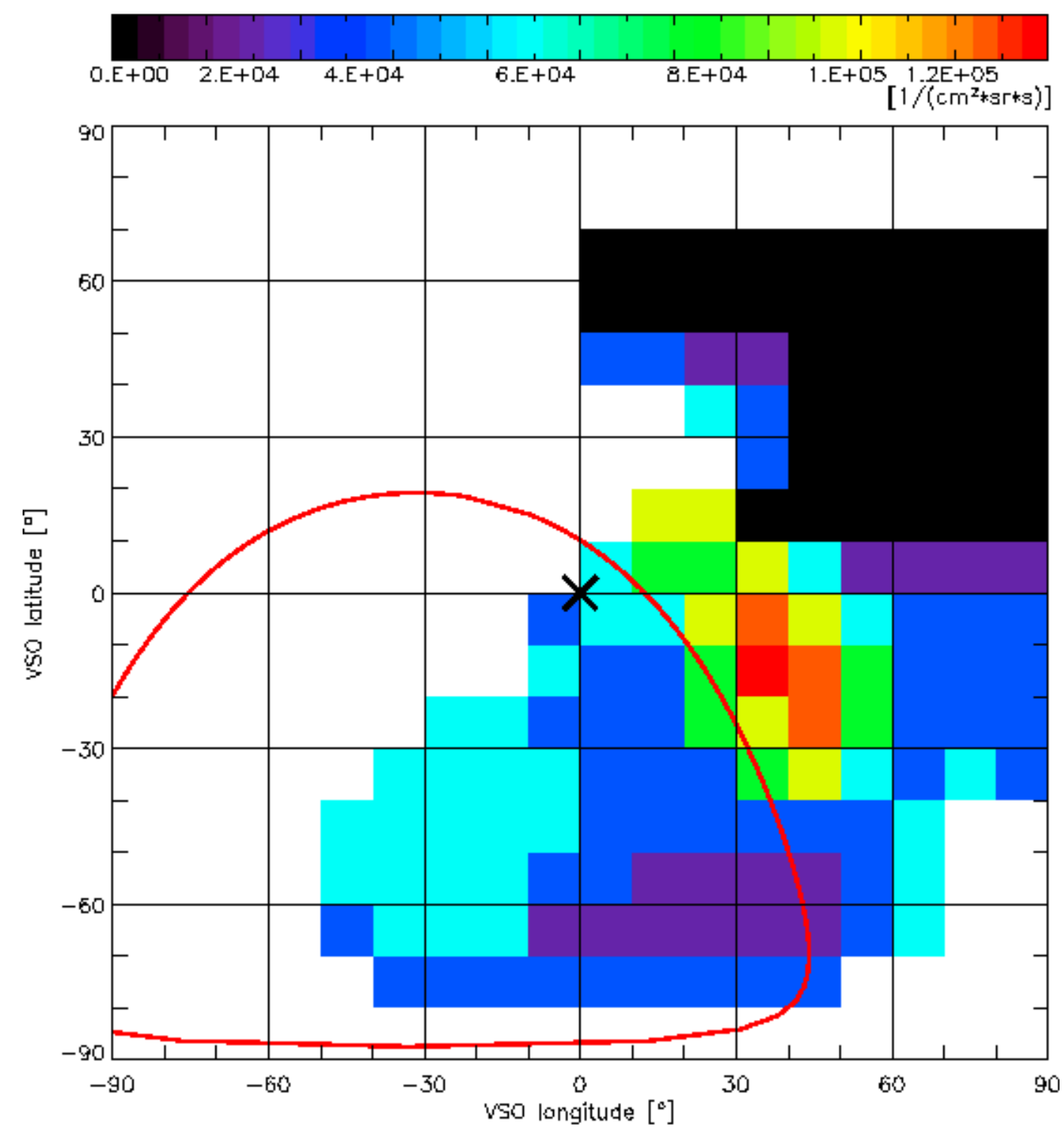

Figure 4. Hydrogen ENA (H-ENA) intensity image 1 of Venus, including 54 measurement intervals from 16 to 29 May 2006. During the measurements the Venus center varies between $-32^{\circ} \pm 10^{\circ}$ longitude and $-34^{\circ} \pm 5^{\circ}$ latitude, the spacecraft altitude is $0.25 \pm 0.05 R_{\mathrm{V}}$. The average FOV footprint equals $38^{\circ} \times 36^{\circ}$

criteria listed in section 3. The data from May to September 2006 resulted in four different images for different vantage points and altitudes of the spacecraft. To create the images, the single intensity measurements were averaged over a $10^{\circ} \times 10^{\circ}$ square mesh. The three most comprehensive images are shown in Figures 4, 5, and 6. The plots are shown in a cylindrical projection of the Venus Solar Orbital (VSO) reference frame, the $x$ axis being the VSO longitude, the $y$ axis being the VSO latitude in degrees. The VSO reference frame is defined as follows: $X_{V S O}$ points from Venus to the Sun, $Z_{V S O}$ points to the North pole of the Venusian orbital plane, and $Y_{V S O}$ closes the right-handed reference frame. By this definition, the Sun's direction is always at $0^{\circ}$ longitude and $0^{\circ}$ latitude. Pixels that are covered more than once by the NPD FOV are shown in color corresponding to the observed ENA intensity. In units of $10^{4} \mathrm{~cm}^{-2} \mathrm{sr}^{-1} \mathrm{~s}^{-1}$, the colors represent the following: black is below 1 , purple is 1 to 2 , blue is 2 to 4 , light blue is 4 to 6 , green is 6 to 8 , yellow is 8 to 10 , orange is 10 to 12 , and red is above 12. The identical color scale is used for observations and for models. Pixels that have not been covered more than once are left white. Because of the fast spacecraft proper motion during the pericenter passage of VEX and because of the simultaneous scanner operations, single measurements are associated with a FOV footprint of typically $40^{\circ} \times 40^{\circ}$. This has to be taken into account when comparing model images with a much higher resolution to the observations (see section 5).

[21] The evaluation of the first and second eclipse season (see list in section 3) yields the following statistics: (1) In 15 of the 16 different orbits the hydrogen ENA signal exceeds the detection threshold. (2) In 14 out of 15 cases the ENA intensity reaches its maximum in the ecliptic plane $\left(-20^{\circ}\right.$ to $\left.+20^{\circ}\right)$ within $40^{\circ}$ around the Sun's direction. 


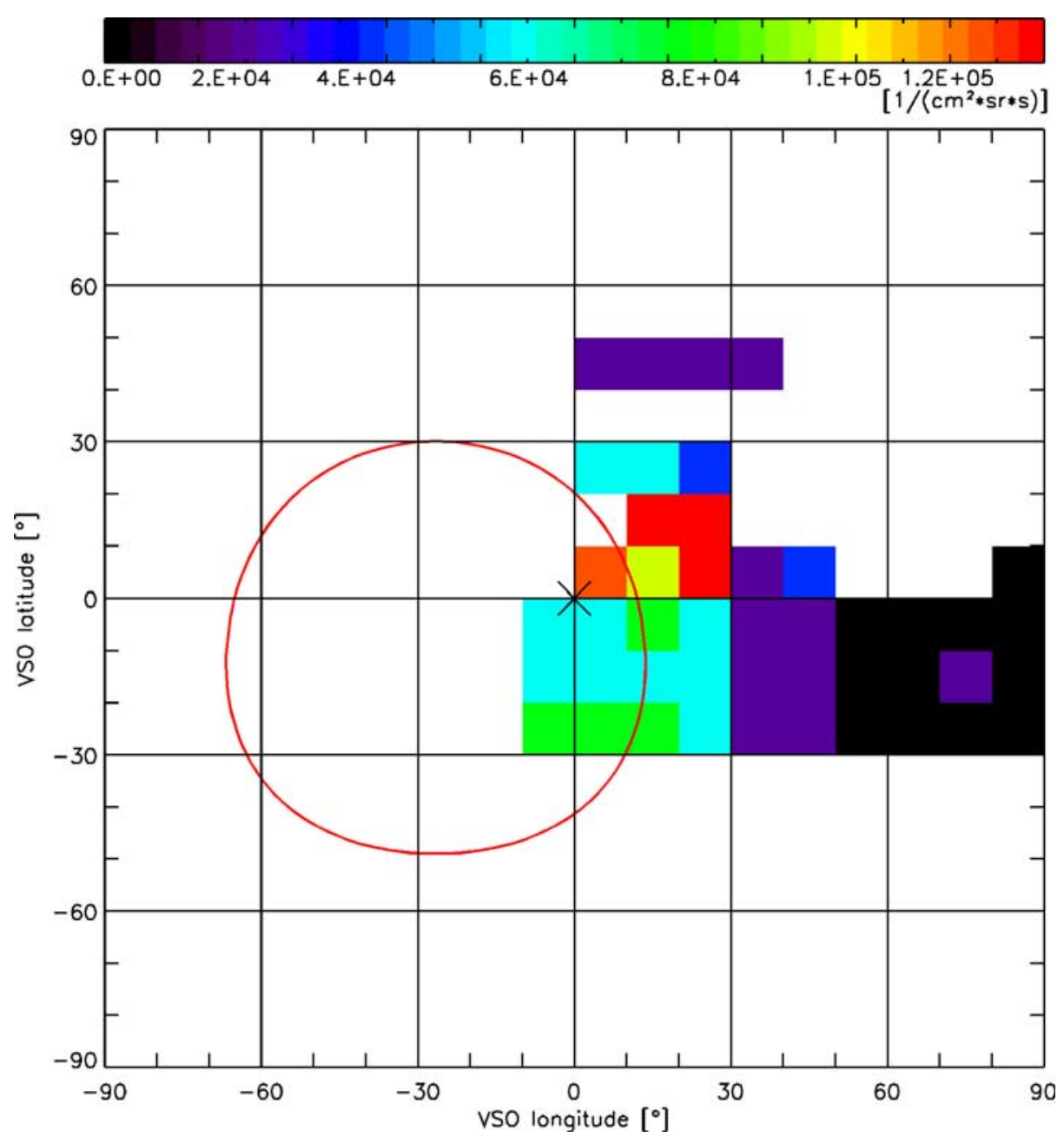

Figure 5. H-ENA intensity image 2 of Venus, including 35 measurement intervals from 16 to 24 May 2006. The Venus center varies between $-27^{\circ} \pm 6^{\circ}$ longitude and $-9^{\circ} \pm 5^{\circ}$ latitude, the spacecraft altitude is $0.57 \pm 0.05 R_{\mathrm{V}}$. The average FOV footprint equals $30^{\circ} \times 30^{\circ}$.

[22] In Figure 4 the maximum ENA outflow seems to be shifted $30^{\circ}$ away from the Sun's direction. However, this shift should not be overinterpreted as it may also be caused by an observational bias: for the image shown in Figure 4 the orange region between $30^{\circ}$ and $60^{\circ} \mathrm{VSO}$ longitude has been covered dozens of times. The region closest to the Sun between $0^{\circ}$ and $30^{\circ}$ VSO longitude, which is predicted to produce even higher ENA intensities, was covered only once.

[23] The median of all 16 orbits calculates to $1.2 \times$ $10^{5} \mathrm{~cm}^{-2} \mathrm{sr}^{-1} \mathrm{~s}^{-1}$ for the direction of most intense ENA outflow; the highest intensity ever measured is $(4.2 \pm 1.6) \times 10^{5} \mathrm{~cm}^{-2} \mathrm{sr}^{-1} \mathrm{~s}^{-1}$. From a direction more than $60^{\circ}$ away from the Venus limb we never (taking into account all NPD observation inside and outside the eclipse) see an unambiguous signal $>4 \times 10^{4} \mathrm{~cm}^{-2} \mathrm{sr}^{-1} \mathrm{~s}^{-1}$.

\section{Comparison to an MHD Model}

[24] To predict the ENA production of a planet, one requires a plasma model for the ion flux distribution around the planet and a model of the neutral exosphere. For Venus only few ENA model calculations have been done so far.
The models presented by Fok et al. [2004] and by Gunell et al. [2005] are technically similar since both are based on MHD calculations of the plasma and PVO measurements of the neutral oxygen and hydrogen exosphere. The resulting maximum ENA intensities on the nightside and the total production rates are consistent with each other within a factor of 2.

[25] To interpret the NPD observations, we use the Venus ENA model developed by Fok et al. [2004]. This model predicts hydrogen and oxygen ENA images based on the MHD plasma simulation by Tanaka and Murawski [1997] assuming the neutral exosphere parameters from the Venus International Reference Atmosphere (VIRA) model [Keating et al., 1985]. A plot of the ion densities, velocities and temperatures calculated by Tanaka and Murawski [1997] is shown in Figure 7. The bow shock is easily recognizable in all three images; the IMB corresponds to the location in the middle where the velocity of the solar wind protons sharply drops from 200 to $0 \mathrm{~km} \mathrm{~s}^{-1}$. No planetary hydrogen ions are included in the MHD model, all predicted hydrogen ENAs (H-ENAs) originate from solar wind protons. The oxygen ENAs (O-ENAs) are caused by accelerated planetary oxygen from the ionosphere (not shown in Figure 7). The most 


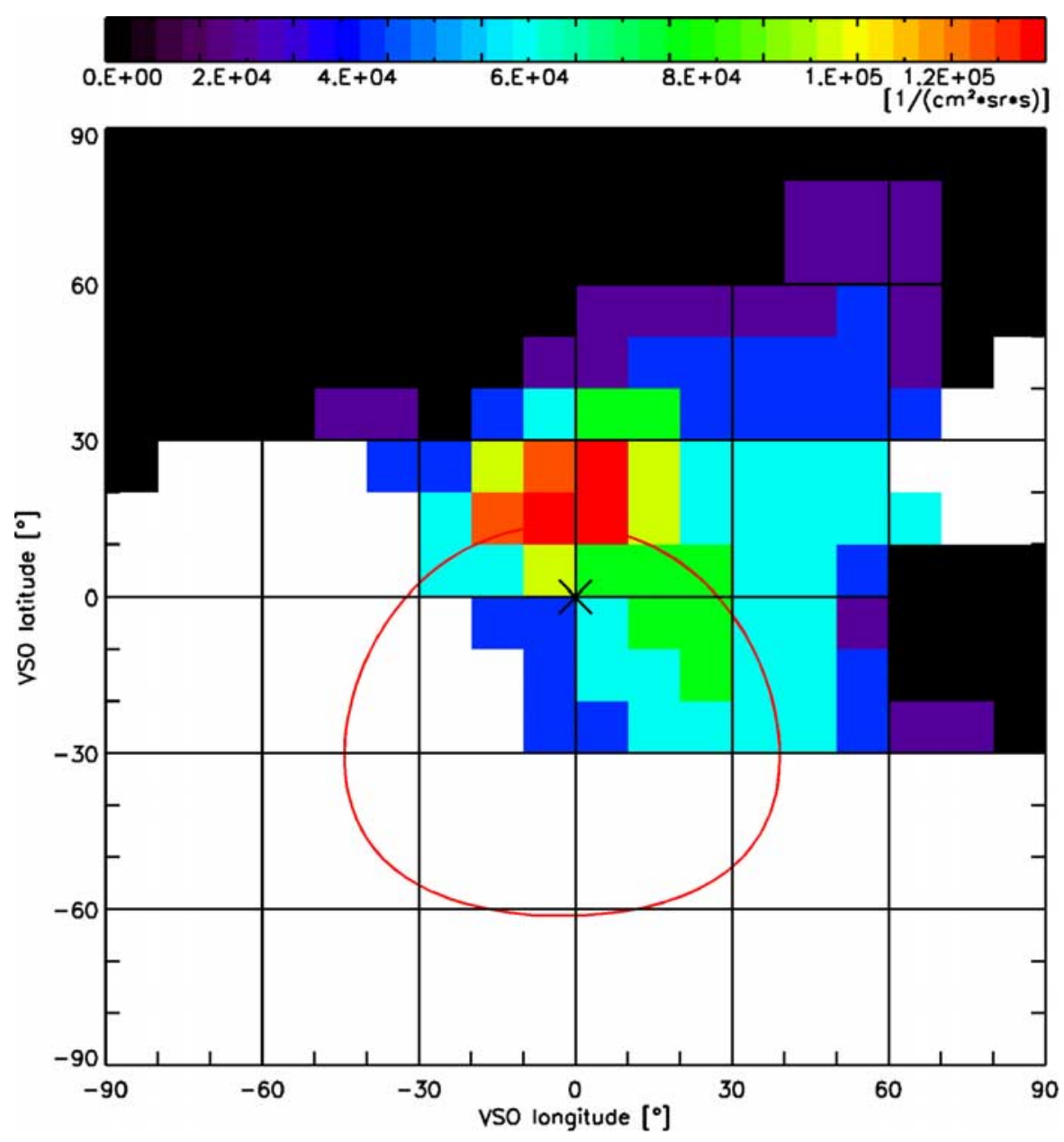

Figure 6. H-ENA intensity image 3 of Venus, including 14 measurement intervals from 20 August to 3 September 2006. The Venus center varies between $-3^{\circ} \pm 12^{\circ}$ longitude and $-24^{\circ} \pm 10^{\circ}$ latitude, the spacecraft altitude is $0.64 \pm 0.20 R_{\mathrm{V}}$. The average FOV footprint equals $70^{\circ} \times 45^{\circ}$.

important model parameters are the solar wind strength and the density profile of the hydrogen exosphere.

[26] The parameters of the exosphere model by Keating et al. [1985] are summarized in Table 1. We implement only the neutral hydrogen and oxygen components, all other species have very low scale heights and particle densities above the exobase. The exobase height is set to $250 \mathrm{~km}$. The vertical density profiles at altitudes above $3500 \mathrm{~km}$, where the VIRA model is not defined any more, are extrapolated using the Chamberlain model [Chamberlain and Hunten, 1987]. If $T$ be the exospheric temperature, $M_{\mathrm{V}}$ the planetary mass, and $r$ the radial distance from the planet center, the scale height $H$ of the density profile is given by

$$
H=\frac{k_{B} T r^{2}}{G m M_{\mathrm{V}}} .
$$

[27] For the thermal hydrogen, equation 2 yields about $200 \mathrm{~km}$ given the temperatures in Table 1, whereas for the hot hydrogen component $H \approx 1000 \mathrm{~km}$. The scale height of the thermal oxygen is only $20 \mathrm{~km}$ at most for the hot subsolar region. Therefore, the exospheric oxygen hardly affects the production of ENAs, and the NPD observations will be sensitive only to the neutral hydrogen parameters listed in Table 1. The only exception to this rule applies to ENA observations at low altitudes above the subsolar region, but this region is never crossed by the LOS of NPD. Above the terminator and the nightside the neutral hydrogen density is higher than the one of oxygen even at the exobase, and the charge exchange cross section for H-ENA production is twice as large as for neutral oxygen [Lindsay and Stebbings, 2005]. Table 1 also indicates that it is the thermal hydrogen component that dominates the ENA production on the nightside. Because of the low exobase density, the particle density of the hot component exceeds the one of the thermal component only at altitudes above $2000 \mathrm{~km}$ according to equation 2 .

[28] As a starting point we will compare the NPD observations to the model predictions using the values in Table 1. Later on we can easily simulate the effects of a thinner or denser neutral exosphere by simply varying the ENA model output. Because we deal with an ENA thin medium around Venus, the predicted ENA outflow depends linearly on the exobase density.

[29] As far as the solar wind is concerned, we have to stick to the parameters implemented in the plasma model by Tanaka and Murawski [1997]: $v_{s w}=400 \mathrm{~km} \mathrm{~s}^{-1}, n_{s w}=$ 

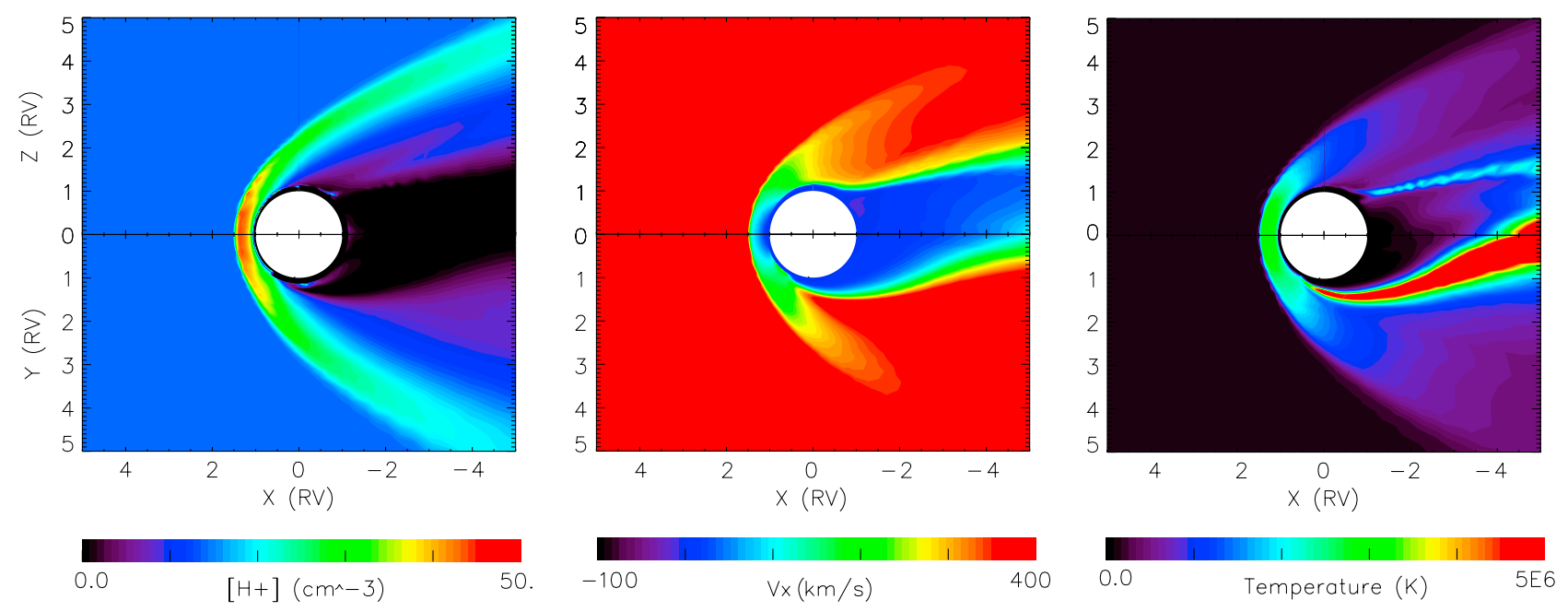

Figure 7. Plasma MHD model by Tanaka and Murawski [1997]. The left shows the proton density, the middle shows the ion velocity along the Sun-Venus line, and the right shows the temperature. The top half corresponds to values in the meridional plane, whereas the bottom half corresponds to values in the equatorial plane of the Venus Solar Orbital (VSO) reference frame. The color scales are linear.

$14 \mathrm{~cm}^{-3}$, and $B_{\mathrm{IMF}}=(0,0,14) \mathrm{nT}$. As mentioned in the introduction, knowledge of $B_{\mathrm{IMF}}$ becomes only important when interpreting ENAs from planetary ions. On the other hand, the intensity of the solar wind ENAs and magnetosheath ENAs directly scales with the flux of the unperturbed solar wind. Unfortunately, we do not know yet the solar wind flux at Venus during the NPD measurements because the absolute calibration of the IMA sensor (and therefore density and speed of the solar wind ions) has not been finished yet. In a recent statistical evaluation of the magnetic field data measured with PVO, Jarvinen et al. [2008] find that the particle flux of $0.6 \times 10^{9} \mathrm{~cm}^{-2} \mathrm{~s}^{-1}$ assumed by Tanaka and Murawski [1997] is close to the most probable flux and is thus a good choice for a single observation. But the median particle flux, observed during solar minimum at Venus, is close to $0.9 \times 10^{9} \mathrm{~cm}^{-2} \mathrm{~s}^{-1}$. The probability that the actual median particle flux encountered during the $16 \mathrm{VEX}$ orbits in 2006 was lower than $0.7 \times 10^{9} \mathrm{~cm}^{-2} \mathrm{~s}^{-1}$ or higher than $1.1 \times 10^{9} \mathrm{~cm}^{-2} \mathrm{~s}^{-1}$ is $25 \%$ in both cases [Jarvinen et al., 2008]. At the end of this section we will discuss how the choice of a solar wind flux that is 1.5 times smaller than the expected median value affects the comparison between model prediction and measurements.

[30] In Figure 8 we compare the ENA intensity spectrum derived from the ENA model to the NPD observation from
24 May 2006. To achieve a direct comparison, we have rebinned the NPD observation shown in Figure 2 (bottom) to the 12 bins between 0.2 and $10 \mathrm{keV}$ used in the ENA model (red curve). Obviously, the modeled integral intensity is higher than the observed one (black curve), around $1 \mathrm{keV}$ the differential intensity is four times higher. The spectral shapes of the NPD measurement and of the model agree in so far as the peak of the model spectrum at $0.8 \mathrm{keV}$ corresponds to a rollover in the NPD spectrum at the same energy. This is the energy of ENAs originating from weakly shocked solar wind. The slopes of the two-component power law that characterizes the measured spectrum seem to be quite different from the model. Note, however, the error bars of the measured spectrum. We find in general that the slopes of NPD energy spectra strongly depend on the instrument efficiency [Grigoriev, 2007] and on the exact algorithm used to reconstruct differential intensities. The position of the rollover and the integral intensity are much more robust parameters. Figure 8 is a further indication that the position of the rollover may be the only trustworthy spectral parameter to be derived from NPD measurements. Moreover, the integration over several minutes blurs all spectral features. We caution the reader against overinterpreting Figure 8. It only indicates that the observed rollover between 0.4 and $2.0 \mathrm{keV}$ (see section 4) is consistent with the hypothesis that the majority of the observed ENA

Table 1. Default Model Parameters of the Neutral Exosphere for Varying Solar Zenith Angle ${ }^{\mathrm{a}}$

\begin{tabular}{|c|c|c|c|c|c|c|c|c|}
\hline SZA (deg) & $n_{\mathrm{H}}\left(\mathrm{cm}^{-3}\right)$ & $T_{\mathrm{H}}(\mathrm{K})$ & $n_{\mathrm{H}, \text { hot }}\left(\mathrm{cm}^{-3}\right)$ & $T_{\mathrm{H}, \text { hot }}(\mathrm{K})$ & $n_{\mathrm{O}}\left(\mathrm{cm}^{-3}\right)$ & $T_{\mathrm{O}}(\mathrm{K})$ & $n_{\mathrm{O}, \text { hot }}\left(\mathrm{cm}^{-3}\right)$ & $T_{\mathrm{O}, \text { hot }}(\mathrm{K})$ \\
\hline 16 & $5.6 \mathrm{e} 4$ & 284 & $1.0 \mathrm{e} 3$ & 1000 & $1.1 \mathrm{e} 7$ & 284 & $6.6 \mathrm{e} 4$ & 4800 \\
\hline 34 & 7.0e 4 & 289 & - & - & $1.1 \mathrm{e} 7$ & 289 & - & - \\
\hline 61 & $2.0 \mathrm{e} 5$ & 292 & - & - & $8.8 \mathrm{e} 6$ & 292 & - & - \\
\hline 90 & $1.1 \mathrm{e} 6$ & 230 & - & - & $1.1 \mathrm{e} 6$ & 230 & - & - \\
\hline 119 & $2.9 \mathrm{e} 7$ & 141 & - & - & $1.2 \mathrm{e} 4$ & 141 & - & - \\
\hline 146 & $1.7 \mathrm{e} 7$ & 124 & - & - & $2.7 \mathrm{e} 3$ & 124 & - & - \\
\hline 164 & $7.5 \mathrm{e} 6$ & 127 & $1.0 \mathrm{e} 3$ & 1500 & $3.0 \mathrm{e} 3$ & 127 & $2.0 \mathrm{e} 3$ & 4800 \\
\hline
\end{tabular}

${ }^{a}$ SZA, solar zenith angle; see Keating et al. [1985]. The most important parameters are the exobase density and the temperature of the thermal hydrogen component. 


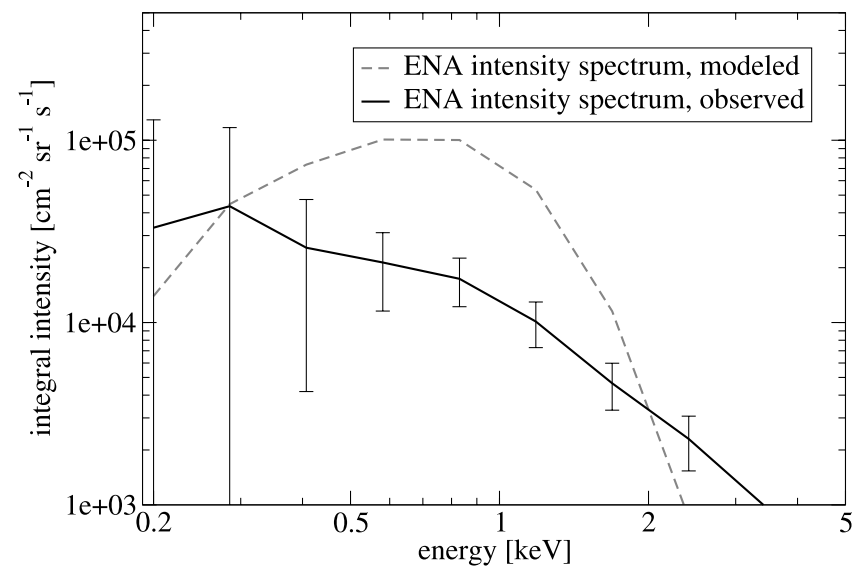

Figure 8. Observed (solid curve) versus modeled (dotted curve) ENA intensity spectrum for the maximum ENA outflow observed on 24 May 2006 (see Figure 2 for the pointing direction).

tailward flow originates from shocked solar wind. Because the derived spectral features are not very reliable except for the rollover, we based the evaluation of NPD observations on the images of the modeled ENA intensities, integrated from 0.2 to $10 \mathrm{keV}$.

[31] We now compare the four ENA intensity images from the first two eclipse seasons (three of them shown in Figures 4, 5, and 6) to the ENA model predictions. The square sum over all image pixels of observed ENA intensity $j_{o b s, i}$ minus predicted ENA intensity $j_{m o d, i}$, divided by the uncertainty of the observed ENA intensity $\sigma_{j, i}$, serves as our merit function:

$$
\chi^{2}=\Sigma_{i}\left(\frac{j_{o b s, i}-j_{m o d}, i}{\sigma_{j, i}}\right)^{2}
$$

The goodness of fit is measured with the probability

$$
P\left(\chi^{2}, f\right)=\gamma\left(0.5 \chi^{2}, 0.5 f\right)
$$

It indicates if the square sum of deviations $\chi^{2}$ between model and observations is tolerable or if the model prediction is very unlikely to be consistent with the observations. As probability threshold below which a model has to be rejected we choose $P\left(\chi^{2}, f\right)=1 \%$. The degree of freedom $f$ is the number $i$ of statistically reliable image pixels (approximately the number of independent measurement intervals) minus the number of model parameters. For the number of model parameters we set 12, appropriate to describe the solar wind strength and the exospheric densities of the thermal and hot hydrogen. Before evaluating equation 3 , the modeled ENA images of $2.5^{\circ} \times 2.5^{\circ}$ resolution are convolved with the same FOV footprints (typically $40^{\circ} \times 40^{\circ}$ ) from which the NPD images were composed. The effect of this smearing process is shown for instance in Figure 10. The typical uncertainties $\sigma_{j, i}$ in equation 3 are as large as $j_{o b s, i}$ : the same location in the image is only covered a few times by independent measurements, each of which with a typical uncertainty of
$30 \%$, and one measurement corresponds to a FOV footprint that covers a dozen image pixels.

[32] Figure 9 shows the ENA model result for the observer position of NPD image 3 (Figure 6). Assuming the original VIRA exobase densities listed in Table 1 the smeared ENA model images show up to $3.5 \times 10^{5} \mathrm{~cm}^{-2} \mathrm{sr}^{-1} \mathrm{~s}^{-1}$ (Figure 9, top). The NPD images, however, do not exceed $1.4 \times$ $10^{5} \mathrm{~cm}^{-2} \mathrm{sr}^{-1} \mathrm{~s}^{-1}$ (red pixels in Figure 6). The corresponding fit probabilities calculated from equation 4 yield $P \geq 1 \%$ for three of the four NPD images. The VIRA model thus is consistent with most NPD measurements but it seems to be an upper limit at low solar activity, at least for the thermal hydrogen component.

[33] Because the default ENA model predicts too high ENA intensities and because we did not have the time to calculate plasma simulations with changed input parameters, we test how a reduction of exospheric densities and temperatures affects the ENA model predictions. We choose two temperature regimes, one as stated in Table 1, the other one with all exospheric temperatures reduced by $20 \%$. This reduction is motivated by the $25 \%$ error bars of the default temperatures suggested by Keating et al. [1985]. That is, an exospheric model with a $20 \%$ lower exobase bulk temperature is a plausible lower limit compared to observations. We then search for the best fitting exospheric density with the crude approach $c \times n_{\mathrm{H}}$ with $c$ one free parameter, identical for all solar zenith angles (SZA). The optimized $c$ is defined by the product of the fit probabilities (equation 4) for the four NPD images. Since most hydrogen ENAs owe their existence to the thermal hydrogen component, the outcome is not changed much whether the correction factor $c$ is applied only to the thermal hydrogen or to all components listed in Table 1 . The resulting ENA intensities change linearly with $c$.

[34] For the default temperatures we find that the agreement between the ENA model and the four NPD images is optimized for neutral hydrogen exobase densities two times lower than in Table 1 . The confidence level of $1 \%$ implies for the range of uncertainty $c=0.5 \pm 0.25$. For $c=0.5$ we find $P\left(\chi_{1}^{2}=43,72-12\right)=96 \%, P\left(\chi_{2}^{2}=45,39-12\right)=1 \%$, but only 2 of 39 pixels deviate from the measurement by more than $2 \sigma_{j, i} . P\left(\chi_{3}^{2}=15,40-12\right)=98 \%, P\left(\chi_{4}^{2}=13\right.$, $46-12)=99.97 \%$, whereby 0 of 46 pixels deviate from the measurement by more than $2 \sigma_{j, i}$. The latter is not a very selective image; because of large error bars and poor spatial coverage it puts only loose constraints on the best fitting model. Obviously, only image 2 (Figure 5) has a really low probability of agreement. This is because of the row of purple pixels to the upper right of the Sun's direction: two independent NPD measurements show for this direction an ENA intensity of $(1 \pm 1) \times 10^{4} \mathrm{~cm}^{-2} \mathrm{sr}^{-1} \mathrm{~s}^{-1}$ whereas the ENA model, convolved with the same FOV footprints, predicts an order of magnitude higher ENA intensities (see Figure 10, bottom).

[35] Two examples for the model with $c=0.5$ are shown in Figures 10 and 11. These images were calculated for the same SZA and planetary distance as the NPD images 2 and 3 in Figures 5 and 6. While the model image with default exospheric densities in Figure 9 has still a fit probability larger than $1 \%$, the fit probability of the ENA model shown in Figure 11 is significantly higher. The model with reduced hydrogen exobase densities is also consistent with the 

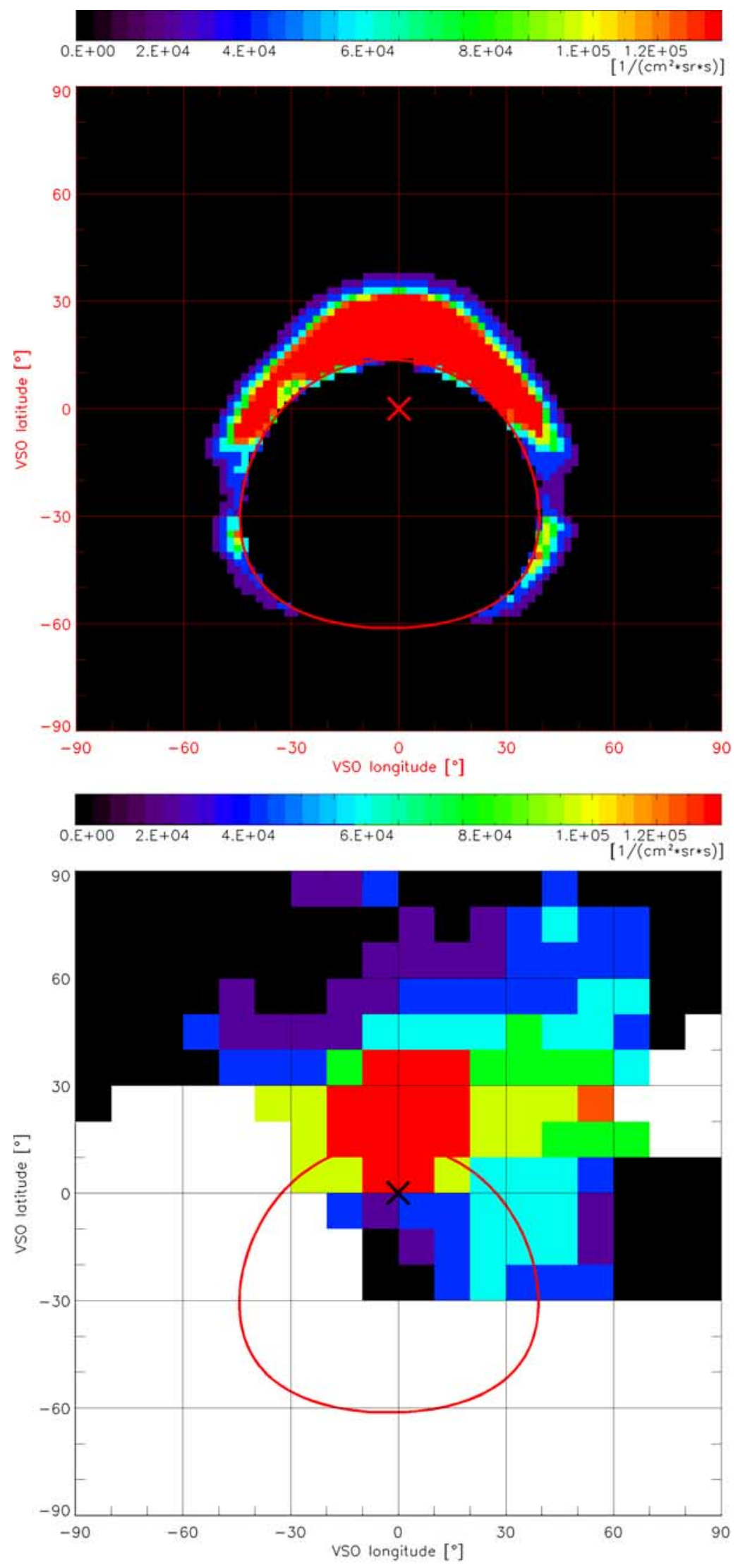

Figure 9. Model prediction of the tailward flow of H-ENAs on the nightside of Venus for image 3 in Figure 6 with the original exospheric densities and temperatures as proposed by Keating et al. [1985]. The top shows the model image in a resolution of $2^{\circ} \times 2^{\circ}$, the bottom is the same image, convolved by the same FOV footprints appropriate for the observed image 3. 

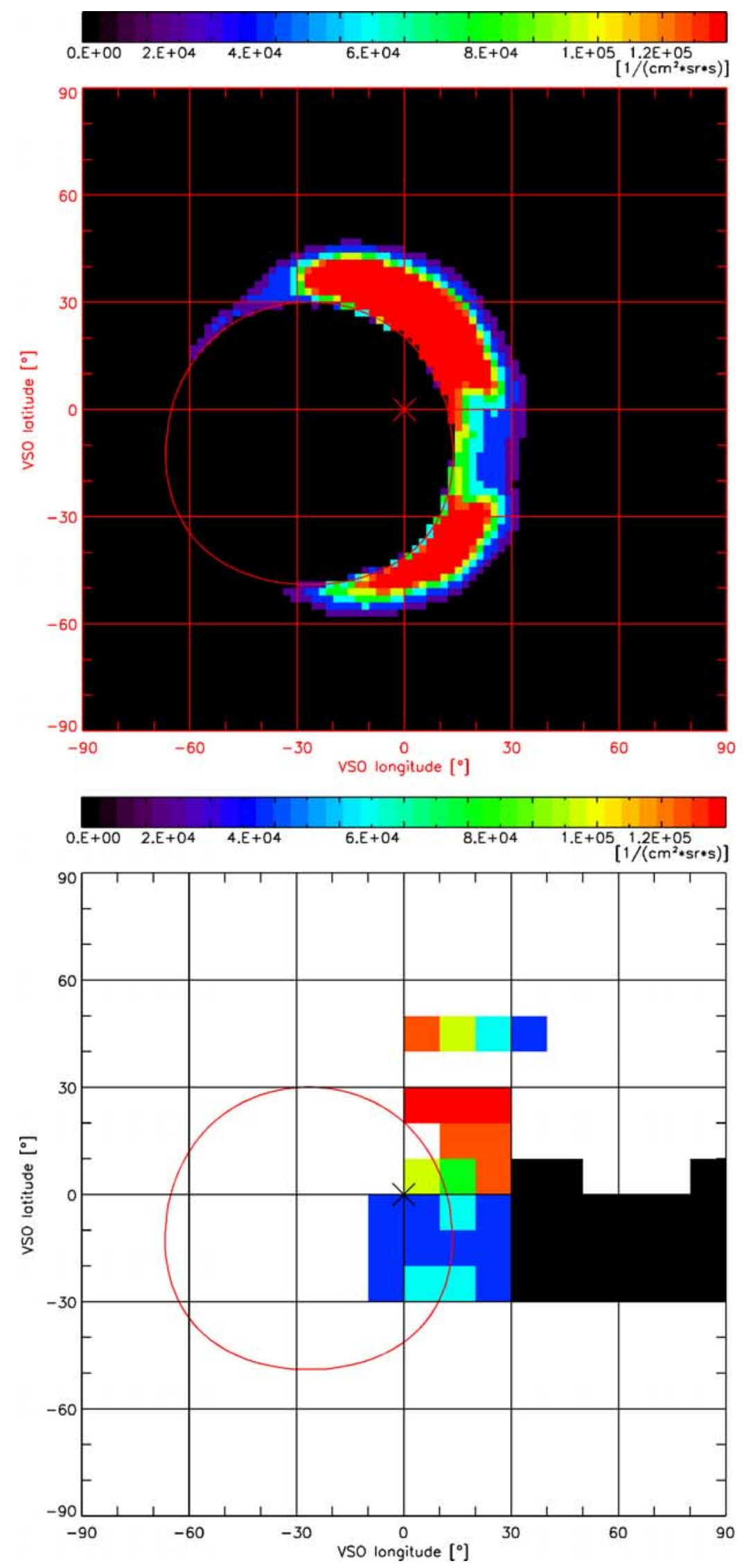

Figure 10. Model prediction of the tailward flow of H-ENAs on the nightside of Venus for image 2 in Figure 5. The hydrogen exobase densities have been reduced by a factor of 2 . The format is the same as in Figure 9. 

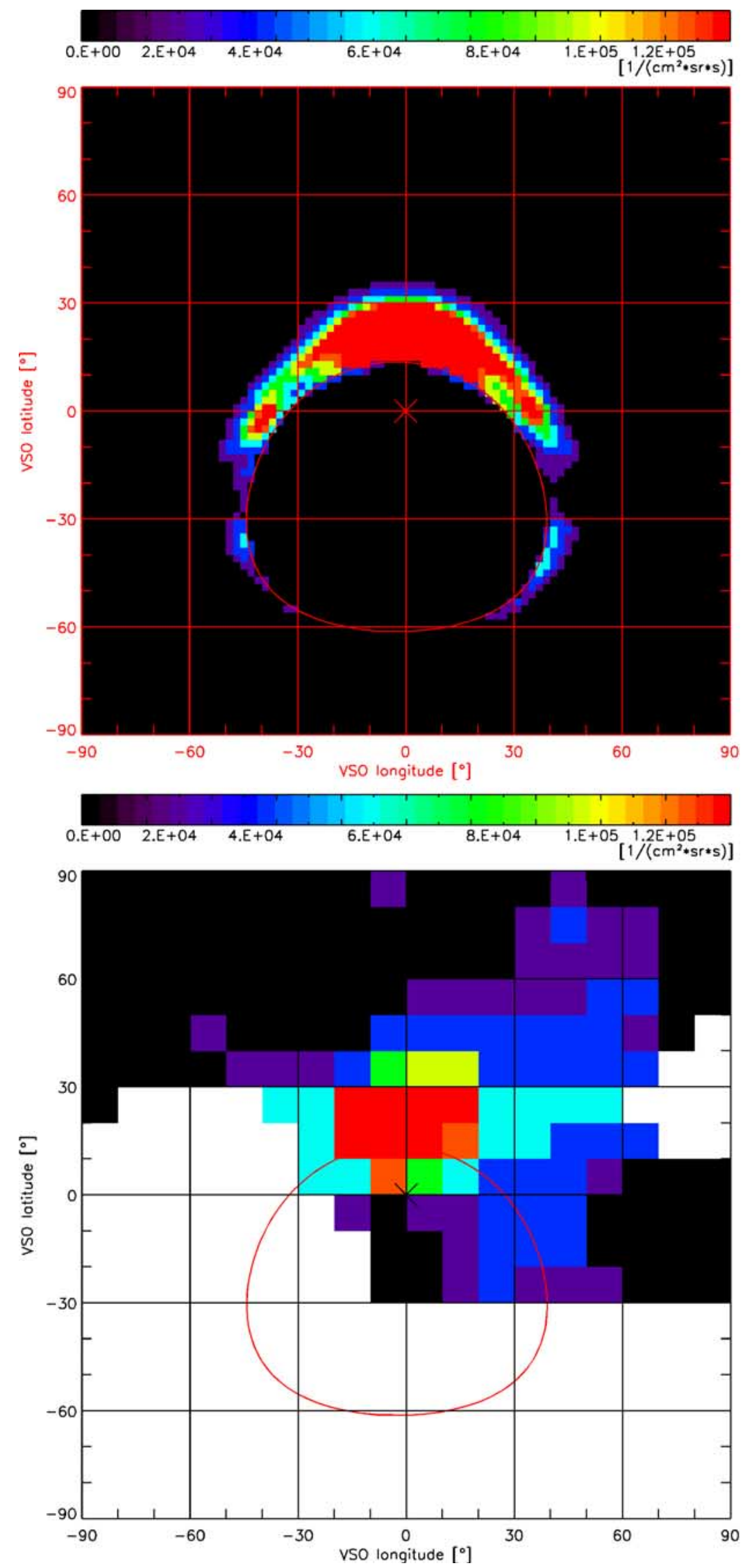

Figure 11. Model prediction of the tailward flow of H-ENAs on the nightside of Venus for image 3 in Figure 6 . The hydrogen exobase densities have been reduced by a factor of 2 . The format is the same as in Figure 9. 

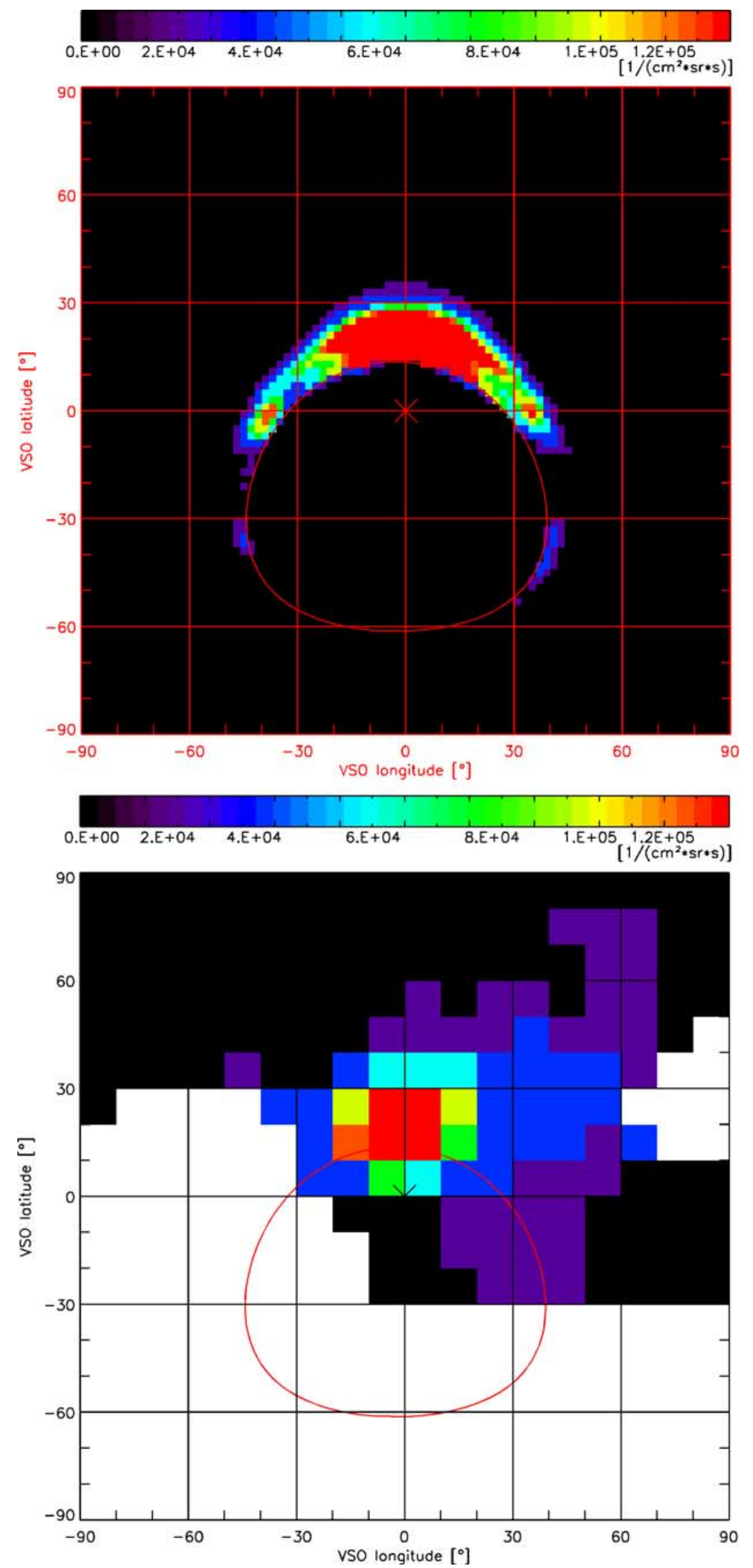

Figure 12. Model prediction of the tailward flow of H-ENAs on the nightside of Venus for image 3 in Figure 6. All exospheric temperatures have been reduced to $80 \%$ of the default values listed in Table 1 . The format is the same as in Figure 9. 


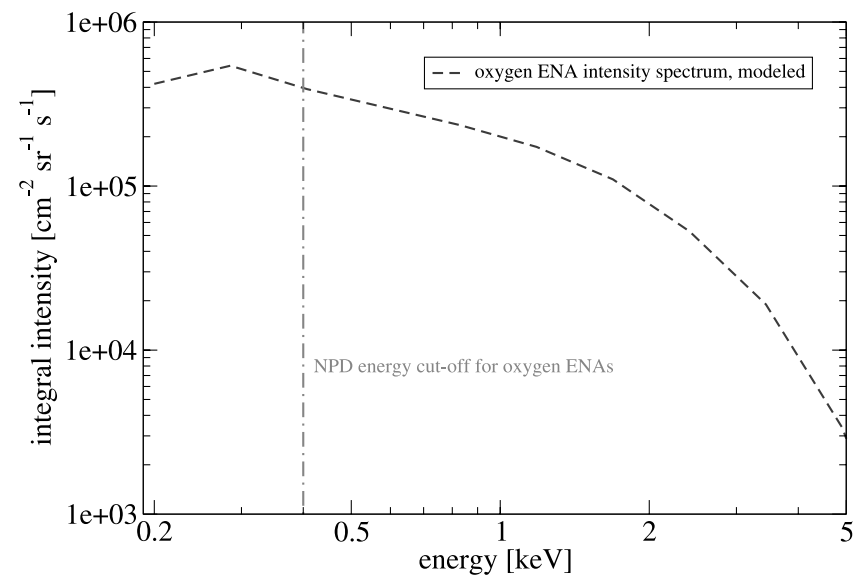

Figure 13. Modeled oxygen ENA intensity spectrum for the pixel of maximum ENA outflow in Figure 14.

observational upper limit on O-ENAs, whereas the original VIRA parameters lead to O-ENA intensities that should be detectable with NPD (see section 6).

[36] Reducing the exospheric temperatures is an alternative possibility to reduce the modeled ENA intensities. Switching on and off the hot hydrogen component has no significant effect on the predicted ENA outflow. The maximum ENA intensities along the Venus limb (the red pixels in Figures 10 and 11) change by $20 \%$ at most. But if the bulk exobase temperatures are reduced by $20 \%$ (including the thermal hydrogen) we find that the predicted ENA intensities drop by a factor of 2. An analogous evaluation as for the models with default exobase temperatures yields a correction factor of $c=1$ with a factor of 2 uncertainty. The range of uncertainty $0.5<c<2$ again corresponds to the confidence level of $1 \%$.

[37] We find that the agreement between the ENA model prediction and the NPD observations is optimized if two times lower hydrogen densities, lower exobase temperatures or a combination thereof is assumed. The low number of independent pixels along the Venus limb and the error bars of the measured images make it impossible to determine which effect offers the better explanation. Comparing Figure 11 (default temperatures, reduced hydrogen densities) to Figure 12 (default densities, reduced temperatures) shows that the two parameter sets lead to very similar ENA intensity images. The only visible difference is that the cool exosphere model leads to intensities that decrease faster for directions away from the maximum ENA outflow.

[38] The only discrepancy between NPD and model images that cannot be solved by adjusting the exospheric parameters is the fact that in two images the observed maximum of the ENA outflow is shifted along the Venus limb away from the Sun's direction. One example of this behavior is shown in Figure 4. Probably it can only be reproduced with a more refined plasma simulation. In all other respects the model images, after smudging them by the NPD FOV footprints, reproduce the four NPD images from the Venus eclipse observations in 2006.

[39] About the influence of the unknown solar wind strength on the model results we can make the following estimate: for low solar activity a median solar wind flux of $(9 \pm 2) \times 10^{8} \mathrm{~cm}^{-2} \mathrm{~s}^{-1}$ is expected [Jarvinen et al.,
2008] whereas the plasma simulation was run for $j_{\mathrm{SW}}=$ $6 \times 10^{8} \mathrm{~cm}^{-2} \mathrm{~s}^{-1}$. In the case of $j_{\mathrm{SW}}=9 \times 10^{8} \mathrm{~cm}^{-2} \mathrm{~s}^{-1}$ the ENA model would predict hydrogen ENA intensities approximately 1.5 times higher than shown here. This would imply that the exospheric densities or temperatures should be reduced even further. Keep in mind, however, that a factor of 2 is usually considered the accuracy in ENA modeling. The model used in this work predicts an ENA production rate that is almost two times higher than the value predicted by Gunell et al. [2005] although the input parameters for the solar wind and the neutral exosphere are identical in both models. Therefore, we do not change the optimized fit parameters for the exospheric hydrogen.

[40] We conclude that the MHD plasma model, including only solar wind protons, reproduces the observed hydrogen ENA intensities and can therefore be relied upon for further work. The observed ENA outflow can be explained as shocked solar wind protons that charge exchange with the neutral hydrogen in the magnetosheath. Hydrogen ENAs of planetary origin are not needed to reproduce the required intensities, and ENAs of unperturbed solar wind protons cannot be observed in the shadow. The high temperature of the shocked solar wind is the reason why NPD detects an ENA signal although the spacecraft is inside the Venus shadow. This can be illustrated with the following comparison: the aperture angle of the ENA outflow around the Sun's direction is observed to be typically $30^{\circ}$ (see Figure 6). We obtain the same result if we refer to Figure 7 for the modeled temperature and flow velocity of the protons inside the magnetosheath at the terminator $\left(T=(2 \pm 1) \times 10^{6} \mathrm{~K}, v=\right.$ $\left.(250 \pm 50) \mathrm{km} \mathrm{s}^{-1}\right)$ and calculate a proton scatter angle of

$$
\alpha=\arctan \sqrt{\frac{2 k T}{m v^{2}}}=25^{\circ} \ldots 50^{\circ} .
$$

[41] To conclude this section, we now calculate the total $\mathrm{H}$ ENA production rate of Venus. The ENA model with default exobase densities and temperatures yields $1.2 \times 10^{25} \mathrm{~s}^{-1}$. Since a hydrogen exosphere with reduced density or temperature fits better to the observations we recommend for low solar activity an H-ENA production rate of $0.6 \times 10^{25} \mathrm{~s}^{-1}$, with a factor of 2 uncertainty. Because the neutral hydrogen exosphere is the dominant neutralizing agent to produce these H-ENAs, the total hydrogen loss of Venus due to charge exchange has to be also at least

$$
Q_{\mathrm{H}, \mathrm{CX}} \geq 0.6 \times 10^{25} \mathrm{~s}^{-1} .
$$

This is consistent with a preliminary evaluation of IMA data [Barabash et al., 2007b] that suggests a lower limit of $10^{25} \mathrm{~s}^{-1}$ for the $\mathrm{H}^{+}$loss through the tail region.

\section{Upper Limit of Observed Oxygen ENAs at Venus}

[42] Observing oxygen ENAs at Venus would be interesting as it would directly reveal an atmospheric erosion process. If there is an observable O-ENA signal it is truly planetary since in the solar wind the oxygen abundance is 5000 times lower than hydrogen. If Venus produces intense O-ENA signals anywhere, they are expected to appear in the 

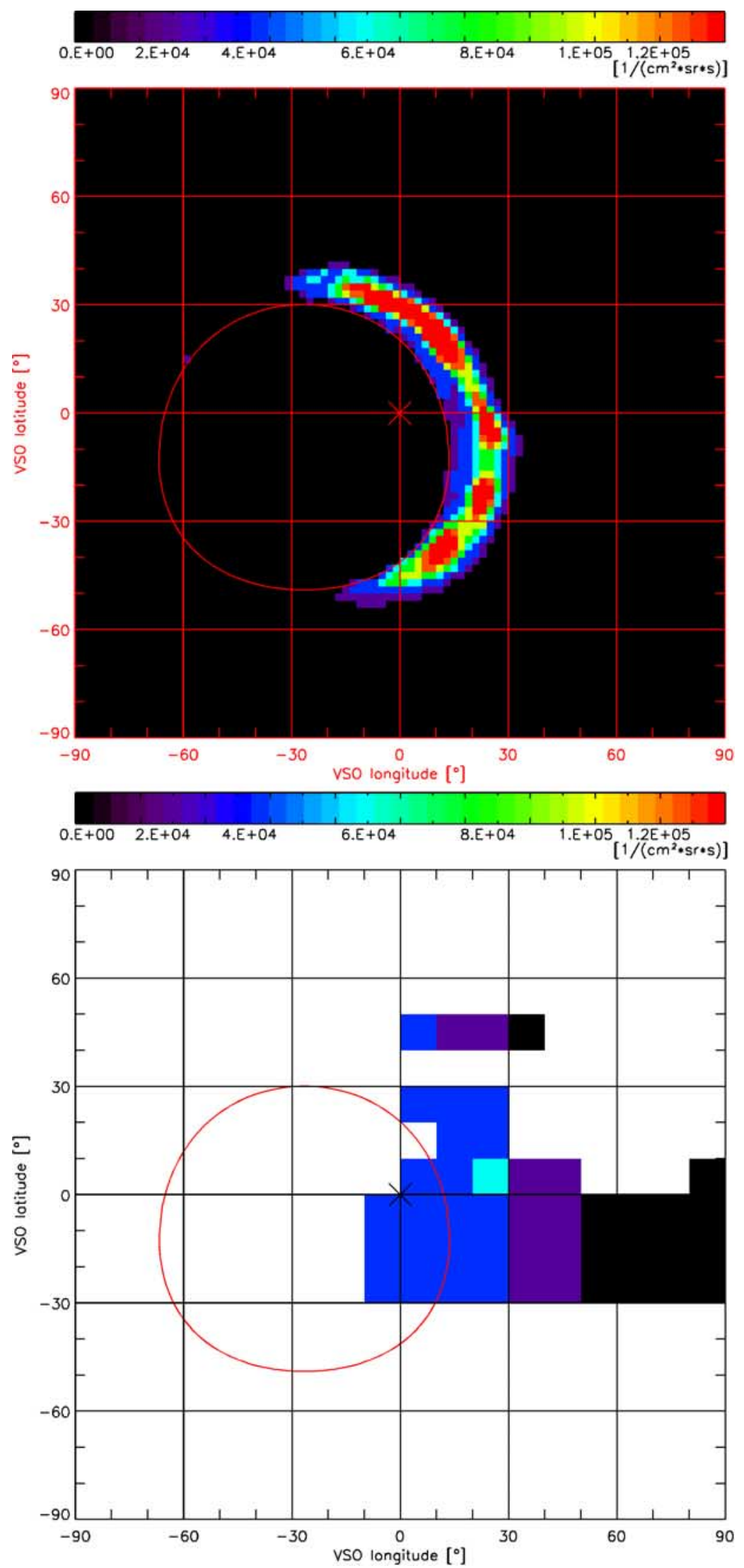

Figure 14. MHD simulation for the tailward flow of O-ENAs on the nightside of Venus for image 2 in Figure 5. The neutral hydrogen exospheric densities have been reduced by a factor of 2 compared to Keating et al. [1985]. The top shows the model image in a resolution of $2^{\circ} \times 2^{\circ}$, the bottom is the same image, convolved by the same FOV footprints appropriate for the observed image shown in Figure 5. 
tailward flow (see Figure 7 by Fok et al. [2004]). According to the calibration in laboratory already an O-ENA signal of more than $1 \times 10^{4} \mathrm{~cm}^{-2} \mathrm{sr}^{-1} \mathrm{~s}^{-1}$ can be observed even in the presence of UV noise and an H-ENA peak if the O-ENA energy lies above $1 \mathrm{keV}$.

[43] The entire data set of Venus eclipse measurements (see list in section 3) has up to now not provided any distinct O-ENA signal. A thorough analysis of the first eclipse season in May 2006, with the laboratory instrument response and efficiency for O-ENAs [Grigoriev, 2007] yields as upper limit for O-ENAs in the tailward flow of Venus the following numbers: $1 \times 10^{4} \mathrm{~cm}^{-2} \mathrm{sr}^{-1} \mathrm{~s}^{-1}$ for energies between 1 and $10 \mathrm{keV}$, and $5 \times 10^{4} \mathrm{~cm}^{-2} \mathrm{sr}^{-1} \mathrm{~s}^{-1}$ for the interval 1.5 down to $0.4 \mathrm{keV}$.

[44] For the default exospheric parameters in Table 1 the model predicts oxygen ENA intensities above the NPD detection threshold. When discussing ENAs originating from planetary ions the ignorance of the $B_{\mathrm{IMF}}$ direction is troublesome (see section 1), but it is very unlikely that during each of the 16 different orbits the convective electric field pointed to a direction not covered with NPD2_2. The median solar wind flux was probably even stronger than assumed in the plasma model, which deepens the discrepancy between model predictions and observations. However, the model predicts oxygen ENA intensities that are consistent with the observed upper limits if two times lower hydrogen exobase densities are implemented (see previous section 5 ). Figure 13 shows the modeled O-ENA spectrum: only 1/3 of the integral intensity is attributed to energies above $0.4 \mathrm{keV}$. If we take that into account and if we reduce the exospheric density of the thermal hydrogen by a factor of 2 , the modeled O-ENA outflow results in Figure 14. The predicted ENA intensities are just below or equal to the observational limit of $5 \times 10^{4} \mathrm{~cm}^{-2} \mathrm{sr}^{-1} \mathrm{~s}^{-1}$, corresponding to the dark blue pixels. Alternatively, reducing the exobase bulk temperature by $20 \%$ also leads to oxygen ENA intensities that are consistent with the observations. The shape of the O-ENA outflow is in general very similar to the H-ENA outflow; it is only slightly narrower. Both the O-ENA and the H-ENA outflow are predominantly caused by charge exchange with neutral hydrogen.

[45] The NPD observations do not rule out that the oxygen exosphere in 2006 was also more tenuous than assumed in the VIRA model. All we can say with the NPD images is that the presented plasma model is consistent with the nonobservation of oxygen ENAs for energies larger than $0.4 \mathrm{keV}$ if we assume hydrogen exobase densities two times lower or exobase temperatures $20 \%$ cooler than the VIRA values. The total O-ENA production rate predicted by the MHD model with reduced hydrogen densities (illustrated in Figure 14) calculates to $7 \times 10^{24} \mathrm{~s}^{-1}$ over the energy range from 0.2 to $10 \mathrm{keV}$.

[46] The total loss of oxygen due to charge exchange reactions at Venus will be higher than the total O-ENA production rate since the loss of oxygen pickup ions that are not neutralized before leaving the exosphere has to be accounted for as well. For the sum of losses due to charge exchange processes Fok et al. [2004] found for the default ENA model $1.5 \times 10^{25} \mathrm{~s}^{-1}$ but they cautioned that this should be understood as a lower limit. The reason is that the MHD model does not take into account finite gyroradius pick up of $\mathrm{O}^{+}$, which will heat up the ionospheric oxygen. On the basis of our current knowledge we estimate the total oxygen loss rate due to charge exchange to be of the order of

$$
Q_{\mathrm{O}, \mathrm{CX}} \approx 10^{25} \mathrm{~s}^{-1} .
$$

\section{Conclusions}

[47] 1. The first ENA images of Venus have been compared to an ENA model. The model, combining the canonical VIRA exosphere model with an MHD plasma code of solar wind ions and planetary oxygen ions, reproduces the observed tailward flow of ENAs within a factor of 2. The observed ENA tailward flow can be understood as neutralized magnetosheath plasma. Although, the uncertainties of the measurements and of the model results limit us to an accuracy of a factor of 2 some exospheric parameters can be constrained.

[48] 2. The MHD approximation seems appropriate to predict the ENA outflow. The resolution of the observed ENA images is insufficient to motivate the use of kinetic models. The images in most cases do not even allow to statistically discriminate the shape of H-ENA outflow against the one of O-ENAs.

[49] 3. The VIRA model by Keating et al. [1985], extrapolated to higher altitudes assuming a Chamberlain profile, is consistent with most NPD measurements. However, the agreement between model and observations is optimized if the exobase densities or temperatures of the thermal hydrogen proposed by Keating et al. [1985] are reduced. If the exobase temperatures are not changed, the NPD observations are reproduced by multiplying the VIRA densities by a factor of $0.5 \pm 0.25$ for all SZA. If the bulk temperatures are reduced by $20 \%$, the possible range for the thermal hydrogen is $1.0_{-0.5}^{+1.0}$ times the VIRA densities. The inherent uncertainties of the observed ENA images do not allow to determine whether the exobase density or the exospheric temperature should be reduced with respect to Keating et al. [1985] for low solar activity. The other neutral components of the Venusian exosphere, including the hot hydrogen component, hardly affect the ENA outflow and therefore cannot be constrained with NPD measurements.

[50] 4. Unfortunately, we cannot say anything about a possible contribution of planetary hydrogen and the influence of the interplanetary magnetic field on the ENA outflow, lacking high spatial and temporal resolution. The present model, which assumes that all H-ENAs originate from solar wind or magnetosheath ions, produces already higher H-ENA intensities than actually observed. Therefore, the contribution of planetary H-ENAs should be of minor importance.

[51] 5. From the model we derive a total hydrogen ENA production rate of $0.6 \times 10^{25} \mathrm{~s}^{-1}$ with a factor of 2 uncertainty. This agrees well with Gunell et al. [2005], who predicted, prior to VEX, an average of $0.7 \times 10^{25} \mathrm{~s}^{-1}$ from a different MHD model. The authors also noted that this number does not vary much over the solar cycle. The H-ENA production rate of Venus is similar to the one measured at Mars with MEX/NPD [Galli et al., 2006b]. For the total oxygen ENA production of Venus at low solar activity an upper limit of $0.7 \times 10^{25} \mathrm{~s}^{-1}$ between 0.2 and $10 \mathrm{keV}$ can be derived. 
[52] 6. We estimate the total loss rates of oxygen and hydrogen due to charge exchange reactions to be of the order of $10^{25} \mathrm{~s}^{-1}$ for low solar activity. This seems to be the same order of magnitude as found in preliminary evaluations of IMA data [Barabash et al., 2007b]. This number will be better constrained in the near future when absolute calibration of the IMA sensor becomes available. Lammer et al. [2006] calculated similar loss rates from ion pickup of $1 \times 10^{25} \mathrm{~s}^{-1}$ for hydrogen and $2 \times 10^{25} \mathrm{~s}^{-1}$ for oxygen. For oxygen this is already close to the total loss rate derived by Lammer et al. [2006]; minor contributions from ion sputtering and detached plasma clouds are predicted to add up to a total of $3 \times 10^{25} \mathrm{~s}^{-1}$. For hydrogen, Lammer et al. [2006] find that the thermal escape of the photochemically produced hot hydrogen component is more important. For this erosion process they predict, on the basis of hydrogen exobase densities of Rodriguez et al. [1984], a loss rate of $4 \times 10^{25} \mathrm{~s}^{-1}$. Rodriguez et al. [1984] studied models with thermal hydrogen densities similar to the default values incorporated in our MHD model. We therefore recommend for the hydrogen loss caused by photochemical processes $Q_{\mathrm{H}, \mathrm{UV}}=(2 \pm 1) \times 10^{25} \mathrm{~s}^{-1}$ for low solar activity.

[53] 7. ENA production on its own is not important for atmospheric loss, neither for oxygen nor for hydrogen. But ENA imaging enables researchers to obtain within short time a global overview on the interaction of the solar wind with planetary atmospheres and ionospheres.

[54] Acknowledgments. The ASPERA-4 experiment on the ESA Venus Express mission is a joint effort between 16 laboratories in 11 countries, all sponsored by their national agencies. We thank all these agencies as well as the various departments/institutes hosting these efforts. The lead author wishes to acknowledge the support of the Swiss National Science Foundation.

\section{References}

Barabash, S., M. Holmström, A. Lukyanov, and E. Kallio (2002), Energetic neutral atoms at Mars: 4. Imaging of planetary oxygen, J. Geophys. Res., 107(A10), 1280, doi:10.1029/2001JA000326.

Barabash, S., et al. (2007a), The analyzer of space plasmas and energetic atoms (ASPERA-4) for the Venus Express mission, Planet. Space Sci., 55,1772 .

Barabash, S., et al. (2007b), The loss of ions from Venus through the plasma wake, Nature, 450, 650.

Bertaux, J. L., J. Blamont, M. Marcelin, V. G. Kurt, N. N. Romanova, and A. S. Smirnov (1978), Lyman-Alpha observations of Venera-9 and $10 \mathrm{I}$. The non-thermal hydrogen population in the exosphere of Venus, Planet. Space Sci., 26, 817.

Chamberlain, J. W., and D. M. Hunten (1987), Theory of Planetary Atmospheres, 2nd ed. Academic, Orlando, Fla.

Coates, A. J., et al. (2008), Ionospheric photoelectrons at Venus: Initial observations by ASPERA-4 ELS, Planet. Space Sci., 56, 802.
Fok, M.-C., T. E. Moore, M. R. Collier, and T. Tanaka (2004), Neutral atom imaging of solar wind interaction with the Earth and Venus, J. Geophys. Res., 109, A01206, doi:10.1029/2003JA010094.

Futaana, Y., et al. (2006a), First ENA observations at Mars: Subsolar ENA jet at Mars, Icarus, 182, 413

Futaana, Y., et al. (2006b), First ENA observations at Mars: ENA emissions from the Martian upper atmosphere, Icarus, 182, 424.

Galli, A., et al. (2006a), Direct measurements of energetic neutral hydrogen in the interplanetary medium, Astrophys. J., 644, 1317.

Galli, A., P. Wurz, S. Barabash, A. Grigoriev, H. Gunell, R. Lundin, M. Holmström, and A. Fedorov (2006b), Energetic hydrogen and oxygen atoms observed on the nightside of Mars, Space Sci. Rev., 126, 267.

Galli, A., et al. (2008), First observation of energetic neutral atoms in the Venus environment, Planet. Space Sci., 56, 807.

Grigoriev, A. (2007), The neutral particle detector on the Mars and Venus Express missions, Ph.D. thesis, Inst. of Space Phys., Kiruna, Sweden.

Gunell, H., M. Holmström, H. K. Biernat, and N. V. Erkaev (2005), Planetary ENA imaging: Venus and a comparison with Mars, Planet. Space Sci., 53, 433.

Gunell, H., M. Holmström, S. Barabash, E. Kallio, P. Janhunen, A. F. Nagy, and Y. Ma (2006), Planetary ENA imaging: Effects of different interaction models for Mars, Planet. Space Sci., 54, 117.

Holmström, M., S. Barabash, and E. Kallio (2002), Energetic neutral atoms at Mars 1. Imaging of solar wind protons, J. Geophys. Res., 107(A10), 1277, doi:10.1029/2001JA000325.

Jarvinen, R., E. Kallio, I. Sillanpää, and P. Janhunen (2008), Hybrid modelling the Pioneer Venus Orbiter magnetic field observations, Adv. Space Res., 41, 1361.

Keating, G. M., et al. (1985), Models of Venus neutral upper atmosphere: Structure and composition, Adv. Space Res., 5, 117.

Lammer, H., et al. (2006), Loss of hydrogen from the upper atmosphere of Venus, Planet. Space Sci., 54, 1445.

Lichtenegger, H. I. M., H. Lammer, and W. Stumptner (2002), Energetic neutral atoms at Mars: 3. Flux and energy distributions of planetary energetic H atoms, J. Geophys. Res., 107(A10), 1279, doi:10.1029/ 2001JA000322.

Lindsay, B. G., and R. F. Stebbings (2005), Charge transfer cross sections for energetic neutral atom data analysis, J. Geophys. Res., 110, A12213, doi:10.1029/2005JA011298.

Martinecz, C., et al. (2008), Location of the bow shock and ion composition boundaries at Venus: Initial determinations from Venus Express ASPERA-4, Planet. Space Sci., 56, 780.

Rodriguez, J. M., M. J. Prather, and M. B. McElroy (1984), Hydrogen on Venus: Exospheric distribution and escape, Planet. Space Sci., 32, 1235.

Tanaka, T., and K. Murawski (1997), Three-dimensional MHD simulation of the solar wind interaction with the ionosphere of Venus: Results of two-component reacting plasma simulation, J. Geophys. Res., 102, 19,805 .

Zhang, T.-L., et al. (2007), Little or no solar wind enters Venus' atmosphere at solar minimum, Nature, 450, 654.

S. Barabash, A. Ekenbäck, Y. Futaana, A. Grigoriev, and M. Holmström, Instituted för Rymdfysik, P.O. Box 812, SE-98128 Kiruna, Sweden.

M.-C. Fok, Geospace Physics Laboratory, NASA Goddard Space Flight Center, Code 673, Greenbelt, MD 20771, USA.

A. Galli and P. Wurz, Physikalisches Institut, Universität Bern, Sidlerstrasse 5, CH-3012 Bern, Switzerland. (wurz@space.unibe.ch)

H. Gunell, Department of Physics, West Virginia University, P.O. Box 6315, Morgantown, WV 26506, USA.

E. Kallio, Finnish Meteorological Institute, P.O. Box 503, FIN-00101 Helsinki, Finland. 